\title{
HISTÓRIA, TEORIA E CRÍTICA NA ARTE CONTEMPORÂNEA: QUESTÕES EMERGENTES DA ARTE-CIENNCIA-TECNOLOGIA
}

\author{
Historia, teoría y crítica en el arte contemporáneo: cuestiones emergentes de \\ arte-ciencia-tecnología \\ History, Theory and Critical in Contemporary Art: Emerging questions of \\ art-science-technology
}

Nara Cristina Santos ${ }^{1}$

\begin{abstract}
Resumo
Este artigo apresenta um percurso em História, Teoria e Crítica na área das Artes Visuais. Busca analisar e discutir questões emergentes de pesquisa transdisciplinar, a partir do projeto Museu Arte-Ciência-Tecnologia: Ações Expositivas e Estratégias Museais (2017-2022), como contribuição para pensar o campo da Arte Contemporânea. Fundamenta-se nas atividades desenvolvidas desde 2007 no PPGART/UFSM, e desde 2005 com a implementação do GP Arte e Tecnologia/CNPq e do LABART, no CAL/UFSM.
\end{abstract}

Palavras-chave: História, Teoria e Crítica; Arte Contemporânea. Transdisciplinar; Arte-Ciência-Tecnologia; Ações expositivas; estratégias museais e arquivos digitais

\section{Resumem}

Este artículo presenta un recorrido en Historia, Teoría y Crítica en el área de las Artes Visuales. Trata de analizar y discutir puntos emergentes de investigación transdisciplinária, desde el proyecto Museo Arte-CienciaTecnología: Acciones Expositivas y Estrategias Museales (2017-2022, cómo contribución para pensar el marco del Arte Contemporáneo. Se fundamenta en las actividades desarrolladas desde 2007 en el PPGART / UFSM, y desde 2005 con la implementación del GP Arte y Tecnología / CNPq y del LABART, en el CAL / UFSM.

Palabras-clave: Historia; Teoría y Crítica; Arte Contemporáneo; Transdisciplinario; Arte-Ciencia-Tecnología. Acciones expositivas; estrategias museales y archivos digitales

\section{Abstract}

This article presents a course in History, Theory and Criticism in the area of Visual Arts. It seeks to analyze and discuss emerging issues of transdisciplinary research, from the project Art-Science-Technology Museum: Expositive Actions and Museums Strategies (2017-2022), as a contribution to think the field of Contemporary Art. It is based on the activities developed since 2007 in PPGART / UFSM, and since 2005 with the implementation of GP Art and Technology / CNPq and LABART, in CAL / UFSM.

Keywords: History; Theory and Criticism; Contemporary art; Transdisciplinary; Art-Science-Technology; Exhibit actions; museum strategies and digital archives

1 - Prof. ${ }^{a}$ Dr. ${ }^{a}$ Associada do Departamento de Artes Visuais (DAV), atua no Programa de Pós-graduação em Artes Visuais (PPGART, Centro de Artes e Letras (CAL) da Universidade Federal de Santa Maria (UFSM), e-mail: naracris.sma@gmail.com 


\section{Percurso, Projetos e Pesquisa no PPGART/UFSM}

Este artigo trata do percurso, do desenvolvimento de projetos e de uma investigação em andamento, intitulada Museu Arte-Ciência-Tecnologia: Ações Expositivas e Estratégias Museais (2017-2022). Busca analisar e discutir questões emergentes de pesquisa, como práticas curatorias, ações expositivas, estratégias museais e arquivos digitais a partir do projeto do Museu, para contribuir para pensar o campo da arte contemporânea. De modo mais abrangente, através da concepção de transdisciplinaridade, presente nas atividades de ensino, pesquisa e extensão do projeto. De modo mais específico, através da área de concentração em Arte Contemporânea, linha de pesquisa Arte e Tecnologia, que envolve tanto os projetos em História, Teoria e Crítica (HTC), quanto em Poéticas Visuais (PV). As atividades são desenvolvidas a partir da atuação como orientadora e professora permanente do Programa de Pós-Graduação em Artes Visuais (PPGART), desde o seu início em 2007, e com a implementação do Grupo de Pesquisa Arte e Tecnologia/CNPq e do Laboratório de Pesquisa em Arte, Tecnologia e Mídias Digitais (LABART), no CAL/UFSM, desde $2005^{2}$.

Figura 1: Site do LABART (http://www.ufsm.br/labart)

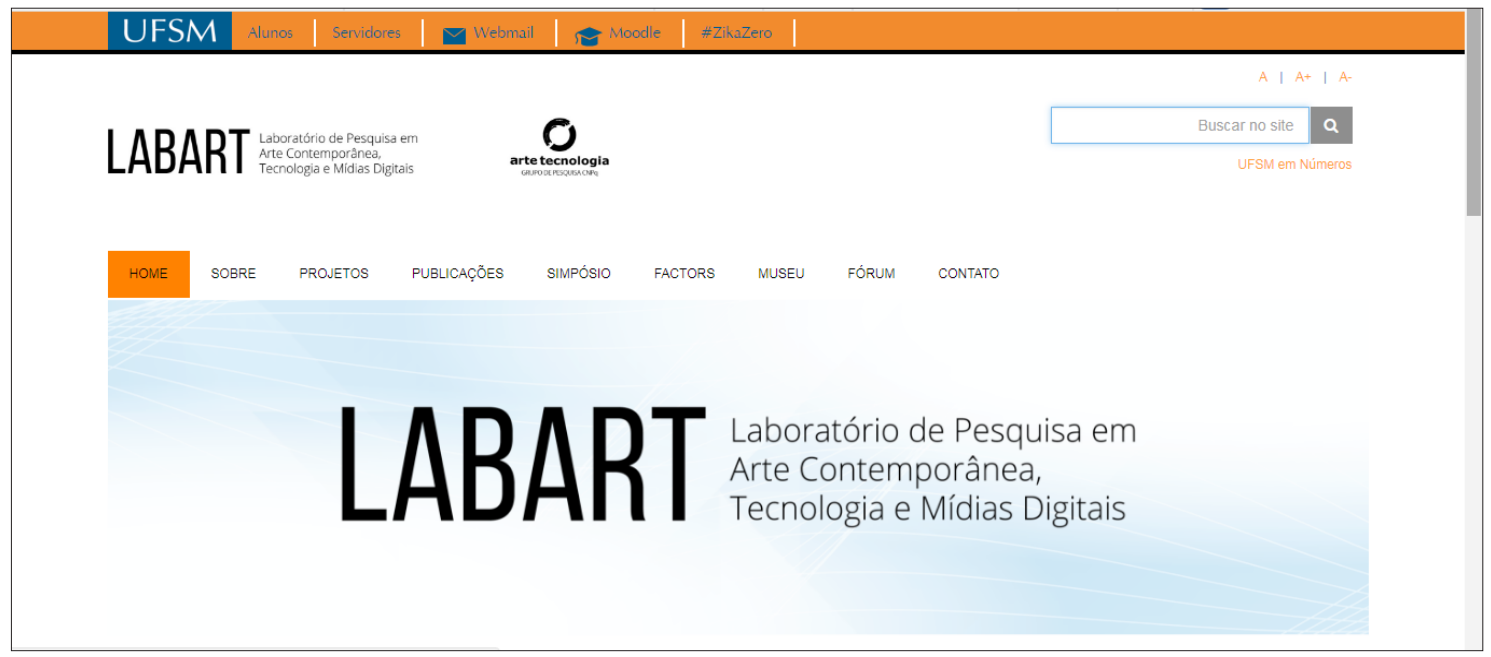

O percurso em HTC pode ser analisado através dos projetos coordenados por esta pesquisadora, vinculados ao PPGART nestes anos, e que, de certo modo, convergem para o atual projeto MUSEU ARTE-CIÊNCIA-TECNOLOGIA: AÇÕES EXPOSITIVAS E ESTRATÉGIAS MUSEAIS (GAP/CAL nº46365 de 2017-2022). Entre eles, outros dois projetos que também apontam uma aproximação inicial da área da museologia são o Museu Interativo: Arte-Ciência-Tecnologia (Ação Neuroarte) (GAP/CAL n 039709 de 2015-2017); e Museu Interativo: Arte, Ciência, Tecnologia e Patrimônio Cultural (GAP/CAL nº29758 de 2011). Entre aqueles que constituem um percurso mais focado na história da arte, estão Artistas Contemporâneos no RS: arte, tecnologia e mídias digitais (GAP/CAL n 024803 de 2009-2013); História da Arte Contemporânea no RS: uma abordagem a partir da produção em arte, tecnologia e mídias digitais (GAP/CAL nº 020625 de 2007-2010); e, Arte, Tecnologia e Mídias Digitais: História da Arte Contemporânea em Ambiente Digital (GAP/CAL nº 017664 de 2005-2010).

Estão vinculadas aos projetos citados, sob orientação desta professora no Mestrado em Artes Visuais, as dissertações de 15 mestres e, seguem em 2018, as pesquisas de 3 mestrandas ${ }^{3}$. Ao projeto do Museu especificamente estão vinculadas

\footnotetext{
2 - http://www.ufsm.br/labart

3 - Mestrandas:

- Natascha Carvalho "Arte e Tecnologia: um estudo sobre as estratégias curatoriais em bienais de arte contemporânea". (2018-)

- Cristina Landerdhal Dalla Costa, "Arte digital na contemporaneidade: desafios para preservação e arquivamento". (2017-)

- Rittieli D’Avila Quiatto, "Espaço expositivo na arte contemporânea: da expografia à mediação em arte e tecnologia”. (2017-)

Mestres:

- Raul Dotto Rosa, "Poética interativa como experiência da presença”. (2018)

- Walesca Timmen Santos, "Vídeo-instalação-interativa: percursos e desdobramentos de universo ficcional”. (2018)

- Andrea Aparecida Capssa de Lima da Silveira, "Considerações sobre as galerias virtuais e suas relações com o mercado de arte”. (2016)

- Valeria Boelter, "Expografia na contemporaneidade: propostas em arte e tecnologia digital". (2016)

- Giovanna Graziosi Casimiro, "Realidade mista e meio expositivo na Arte Contemporânea: insitu<>influxu”. (2015)

- Carlos Alberto Donaduzzi, “Fotografia submersa: cenas cotidianas ficcionais". (2014)

Manoela Freitas Vares, "Ciborgue: uma concepção do corpo na Arte Contemporânea". (2013)

- Anelise Vieira dos Santos Witt, "Gamearte: subversão e diversão na Arte Contemporânea". (2013)

- Débora Aita Gasparetto, "Arte digital e circuito expositivo: um "curto em torno do FILE". (2012)

Henrique Telles Neto, "Interdisciplinaridade em arte, ciência e tecnologia: SCIARTS". (2012)
} 
3 dissertações defendidas e outras 3 em andamento. Neste percurso, oportunizou-se aos mestrandos, a partir destes projetos, o desenvolvimento de suas investigações com bibliografias atualizadas, em contato com artistas e pesquisadores reconhecidos na área das Artes Visuais, e de Arte, Ciência e Tecnologia (ACT); assim como incentivou-se a participação dos mestrandos para apresentação de trabalhos em congressos e exposições nacionais e internacionais, para publicação, e na organização de eventos junto ao LABART. Estes fatores permitiram a cada um deles desenvolver sua pesquisa de mestrado com um foco específico em arte e tecnologia, abordadas no campo da arte contemporânea, trazendo uma efetiva contribuição para história, teoria e crítica da arte e/ou significativas produções artísticas em poéticas digitais. Em 2018 , dos 15 mestres orientados em 10 anos, 9 são professores em Instituições de Ensino Superior (IES); entre os 15, 5 são doutores e outros 3 estão em doutoramento, em outras IES no Brasil e em Portugal.

\section{Para fundamentar a pesquisa em HTC e ACT: algumas referências}

Para dar conta, no campo da história e teoria da arte, das bases de pesquisa para o projeto do Museu, os historiadores brasileiros Walter Zanini e Daisy Piccinini são fontes exemplares. Alguns autores internacionais, entre outros, relevantes para o trabalho desta pesquisadora, são: Hans Belting que trata da história da arte (e seu fim) e da história das mídias; Edward Shanken da história da arte e da tecnologia a partir da concepção de sistema; e Oliver Grau, que discorre sobre a história da arte mídia, a arte virtual e as instituições culturais na era digital, museus e processos de arquivamento. Somamse a estes, Terry Smith que contextualiza a história da arte contemporânea no âmbito da curadoria, instituições e mercado; Boris Groys que discute a reprodução mecânica e digital e as práticas culturais no âmbito da Internet. Também, Didi-Hubermann, nas publicações que compartilha a atuação de historiador, crítico e curador, incluindo a mostra Sublevaciones; Hans Obrist, com seus estudos e práticas curatorias; Jérôme Glicenstein, sobre a atuação do curador na arte contemporânea; Jacqueline Eidelman, tratando do público dos museus; Catherine Grenier, apontando as crises institucionais dos museus; Dominique Poulot, discorrendo sobre o campo da museologia; Mariana Salgado, tratando de como desenhar um museu aberto. Ainda, Cristiane Paul, sobre as especificidades de um espaço expositivo para Arte Digital; Peter Weibel, para pensar arte contemporânea e museu, na experiência do ZKM; Stephen Wilson, discorrendo nas interseções entre arte, ciência e tecnologia; e, autores mais específicos para cada uma das ações expositivas do Museu, como por exemplo Daniel Rincón, que fundamentou o argumento curatorial para a mostra Bioarte. Estes são apenas alguns referenciais, entre tantos outros internacionais e nacionais.

Na pesquisa em história teoria e crítica na arte contemporânea, mais precisamente em arte e tecnologia digital, a compreensão do conceito de arte como sistema artista<> obra<>interator <>entorno <>contexto ${ }^{4}$ colabora para compreender as produções transdisciplinares em arte, ciência e tecnologia. Nessa concepção de arte como sistema, não é um problema entender o contexto da arte contemporânea no âmbito institucional, incluindo os espaços de exposição e as próprias ações expositivas.

As ações anteriores fundamentam este projeto mais recente MUSEU ARTE-CIÊNCIA-TECNOLOGIA: AÇÕES EXPOSITIVAS E ESTRATÉGIAS MUSEAIS como ensino, pesquisa e extensão. No ensino, aborda-se tanto as produções de monografias de curso de graduação quanto as dissertações de mestrado; na pesquisa, discute-se a investigação sobre artistas e obras em arte ciência tecnologia, o estudo do espaço expositivo, das estratégias museais e arquivos digitais; na extensão, englobamse eventos como o Simpósio de Arte Contemporânea, o FACTORS (Festival de Arte, Ciência e Tecnologia do RS), as ações expositivas, que se dividem em artísticas e modulares, entre outras atividades.

O projeto pensado para cinco anos estabelece uma espécie de fechamento das pesquisas iniciais, de um caráter mais teórico, para uma atuação também teórico-prática-teórica, quando busca uma aproximação mais direta na relação com artistas, obras e o público. Também permite uma constante atualização em torno das mostras e exposições que aconteceram antes e, de certo modo, foram uma preparação desde 2005 para a convergência de um festival. Com o projeto do Museu abrangendo o FACTORS como uma ação expositiva desde 2016, e em 2017 integrando a BIENALSUR, teve-se um importante reconhecimento não apenas pela internacionalização atingida, mas para reafirmar a necessidade de um espaço expositivo institucional que possa vir a se concretizar como um Museu de fato, o MACT - Museu Arte-Ciência-Tecnologia da UFSM.

Então, pode-se perguntar: como um projeto transdisciplinar no campo da arte, da ciência, da tecnologia aliado à história, teoria e a crítica, pode estabelecer alguma diferença em HTC para o campo da arte e da cultura? Sem dúvida, no amadurecimento das ações expositivas; no estudo e na elaboração de argumentos mais consistentes para curadorias em

\footnotetext{
Fernando Franco Codevilla, “Video-perfomance: processos com o audiovisual em tempo real”. (2011)

Greice Antolini Silveira, "Imersão: sensação redimensionada pelas tecnologias digitais na Arte Contemporânea". (2011)

Cláudia Loch, "No labirinto da urbis ao ciberespaço: uma poética digital". (2010)

- Fabiane Sartoretto Pavin, "Processos Híbridos na Poética de Sandra Rey: um estudo a partir de 'Soft Dreams' e 'Desdobramentos da Paisagem”'. (2010)

Franciele Filipini dos Santos. "O ciberespaço e o ambiente virtual da Bienal do Mercosul: possível espaço de criação/exposição". (2009)

4 - SANTOS, Nara Cristina. Arte (e) Tecnologia em sensível emergência com o entorno digital. Tese de Doutorado em Artes Visuais/UFRGS, 2004.
} 
arte, ciência e tecnologia; na dedicação ao projeto expográfico mais adequado às especificidades do espaço, às demandas de artistas e às obras transdisciplinares, e na possibilidade de interação do público; na definição e uso dos espaços para exposição, a partir da aceitação e da adaptação da arquitetura disponível, das condições técnicas, tecnológicas, também de temperatura, ventilação, umidade e iluminação; no desenvolvimento de estratégias museais que aos poucos se consolidam, e que incluem pensar em acervo, manutenção e arquivamento digital.

Nessa caminhada iniciada a partir de 2011, com o primeiro projeto do MACT, a exposição realizada da artista Anna Barros, com a obra resultante da pesquisa em nanoarte, foi sem dúvida a melhor experiência antes da ação de 2016, do FACTORS 4.0, que reuniu artistas e obras dialogando com a bioarte. Melhor pela integração das áreas envolvidas, pela proposição crítica das questões conceituais, pelo espaço do MASM-Museu de Arte de Santa Maria, e porque a obra da Anna depois se tornou a primeira do acervo do MACT (um museu sem espaço físico fundado nas ações expositivas em espaços institucionais). É a primeira obra do acervo, toda tecnológica, e também aquela que nos desafia, pois está aberta para um estudo real de manutenção e arquivamento.

Nesse sentido as questões lançadas na concepção da arte como sistema apontam tanto inter-relações teóricas, quanto teórico-práticas para entender cinco momentos, "criação, produção, visualização, disponibilização e manutenção" ${ }^{5}$, que propiciam analisar obras contemporâneas. Sejam elas resultados de relações da arte e ciência, arte e tecnologia, arte e ciência e tecnologia, todas são produções artísticas, contribuindo para ampliar o campo da arte e da cultura, aliado ao meio ambiente e à sustentabilidade, para citar apenas um viés transdisciplinar.

\section{Projeto Museu Arte-Ciência-Tecnologia: Ações Expositivas e Estratégias Museais ${ }^{6}$}

O Projeto Museu Arte Ciência Tecnologia de caráter transdisciplinar, tem permitido, a partir da área de investigação nas Artes Visuais, estudar artistas e obras em poéticas digitais, discutir questões emergentes no campo da arte, ciência e tecnologia digital, aprofundar o estudo e a prática em curadoria e expografia, mais especificamente sobre ações expositivas e estratégias museais, para contribuir com a História, Teoria e Crítica da Arte Contemporânea. Considerando a formação na área das Artes Visuais e a trajetória desta pesquisadora em arte, ciência e tecnologia, mais recentemente se está mantendo pontos de contato com algumas questões da área da museologia, para contextualizar a abrangência deste projeto.

Figura 2 - Site do Museu Arte Ciência Tecnologia (http://www.http://museuarteciencia.ufsm.br)

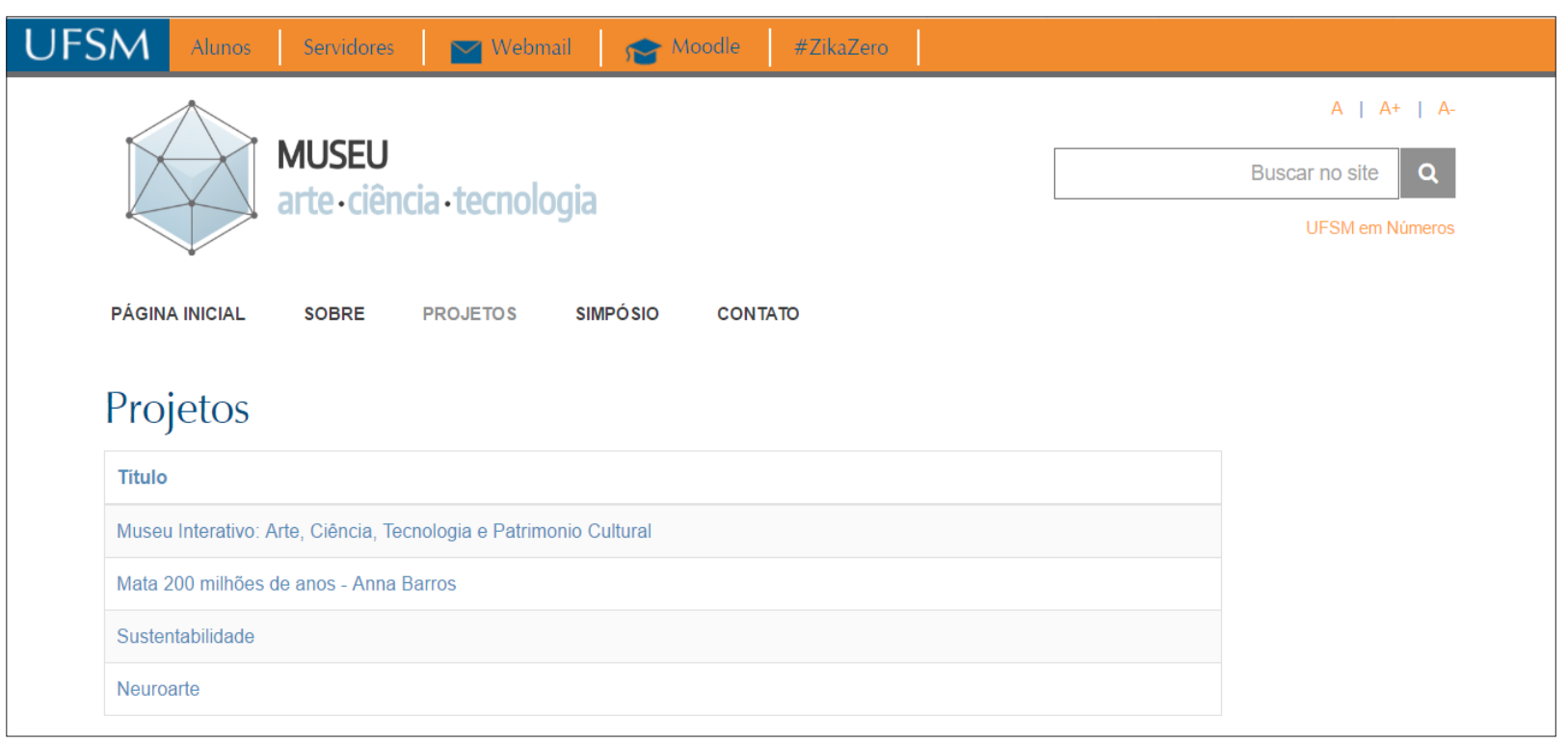

5 - SANTOS, Nara Cristina. Arte (e) Tecnologia em sensível emergência com o entorno digital. Tese de Doutorado em Artes Visuais/UFRGS, 2004.

6 - SANTOS, Nara Cristina. Museu Arte-Ciência-Tecnologia: artistas em ações transdisciplinares, p.442-453. In: Diálogos Transdisciplinares: Arte e Pesquisa. PRADO. G; TAVARES. M; ARANTES. P. (Org). São Paulo: ECA/USP, 2016.

- SANTOS, Nara Cristina. Museu Arte Ciência Tecnologia: um projeto, seu percurso e seus percalços, p.208-220. In: Configurações do pós-digital: Arte e cultura tecnológicas. GOBIRA, P.; MUCELLI, Tadeus (Org). Belo Horizonte: EdUEMG, 2017.

- SANTOS, Nara Cristina. História da Arte e Transdisciplinaridade: ação expositiva do projeto Museu Arte Ciência Tecnologia. ANAIS CBHA 2017. Salvador: EdUFBA, 2018. 
Nesse sentido, pensar o Museu através de ações expositivas e estratégias museais parece compreensível. O projeto do museu interativo começou em 2010, a partir de duas pesquisadoras ${ }^{7}$, coordenadoras de cursos de pós-graduação na época, interessadas em propostas transdisciplinares que pudessem ser exploradas na articulação do ensino, pesquisa e extensão através de um espaço expositivo. Junto aos respectivos programas, o PPGART/Artes Visuais e o PPG Educação para Ciência, articularam-se os PPGS em Ciências Sociais, Informática e Patrimônio Cultural, em um projeto comum, para construção do que na época denominou-se "Museu Interativo - Arte, Ciência, Tecnologia e Patrimônio Cultural", com apoio de arquitetos da UFSM, professores e alunos do Curso de Arquitetura.

Em 2016, redefiniu-se o projeto para Museu Arte Ciência Tecnologia ${ }^{8}$. Primeiro, porque constatou-se neste percurso que a interatividade nem sempre seria o foco das exposições, mas também a interação e a participação. Segundo, porque estas são de fato as três áreas de conhecimento que, integradas, permanecem desde 2012 trabalhando juntas. Nestes anos, ainda sem espaço físico próprio, realizamos diferentes ações expositivas e, na área das Artes Visuais, contou-se com a presença de artistas reconhecidos nacional e internacionalmente, assim como artistas emergentes nas seguintes ações: no MASM, 2011 - "Mata-200 milhões de anos Árvore Pedra", com Anna Barros; na UFSM, 2013 - "Arte-Sustentabilidade-Ciência", com Guto Nóbrega, Malu Fragoso, Carlos Donaduzzi, Fernando Codevilla e Anelise Witt; no CAL/UFSM, 2015 - "Neuroarte", com Alberto Semeler; no CAL/UFSM, 2016 - "Neurociência e Arte: percepção sensível", com Mariela Yeregui, Manuela Lopez, Raquel Zuanon, Rosangela Leote, Tania Fraga, entre outros (junto ao FACTORS 3.0); e, no CAL/UFSM, 2016 - ação "Bioarte", reuniu os artistas nacionais Fernando Codevilla e Leonardo Arzeno, Gilberto Prado, Guto Nóbrega, Rebeca Stumm, Walesca Timem e Raul Dotto, e Yara Guasque; e, o brasileiro que vive em Chicago, Eduardo Kac; e, internacionais, entre os quais as argentinas Paula Guersenzvaig, Ana Laura Cantera e o Grupo Robô Mestizos; e, as mexicanas do Grupo Electrobiótas, Gabriela Lílian Munguía Ortíz e Guadalupe Chávez Prado (junto ao FACTORS 4.0, integrado à BIENALSUR).

Estas ações expositivas, somadas a atualização do conhecimento nas áreas envolvidas, desencadearam um percurso próprio e uma dinâmica expositiva que deu ao grupo, formado por pesquisadores, artistas, estudantes de pós-graduação e graduação, e técnicos, consistência e competência. O reconhecimento dos resultados, com a maturidade nesta fase inicial, foi alcançado em 2014 com a aprovação do projeto NeuroArte: Museu Itinerante de Neurociência, Arte e Tecnologia pela Chamada MCTI/CNPq/SECIS n.085/2013, apoio à criação e ao desenvolvimento de centros e museus de ciência. ${ }^{9}$

O projeto do MACT se interroga sobre a concepção de museu, ao gerar um espaço de confluências, divergências e conexões para a produção de conhecimento artístico, científico, tecnológico e cultural em torno de um argumento crítico a cada ação transdisciplinar. Nesse sentido, a transdisciplinaridade propicia aos pesquisadores o compartilhamento, as conexões e as redes para produção de conhecimento complexo. Portanto, compreende-se a prática transdisciplinar não apenas como um modo de organizar conhecimento a partir de diferentes disciplinas para constituir um pensamento sistêmico, mas também como um modo de se deixar atravessar, na produção artística, por uma ação complexa. O pesquisador na área da arte 'parte de seu lugar' e estabelece um vínculo com o pesquisador de outra área, cujo trabalho, processo e resultado da investigação apontam questões que não pertencem mais a nenhuma das áreas, mas ao que se constitui para além delas, um campo de conhecimento transdisciplinar em constante emergência. Experiências transdisciplinares e compartilhadas como estratégias político-culturais são pretensões do projeto do Museu.

O projeto que começava com a pretensão de um espaço expositivo, torna-se um projeto de ações expositivas. Fundado em ações transdisciplinares, de caráter temporário, o projeto vem sendo fomentado por incentivos públicos através de apoios institucionais, através de editais, parcerias entre instituições e equipamentos culturais públicos. Conta com infraestrutura expográfica, técnica, tecnológica, informacional, arquivista e de pessoal, dos laboratórios envolvidos e da UFSM.

De certa maneira, o desenvolvimento desse projeto do Museu surge como agregador de experiências anteriores e muito provavelmente aberto a outros projetos de ensino, pesquisa e extensão para investigações futuras. Um determinado patamar foi atingido de responsabilidade, compromisso institucional e de retorno para a comunidade, a partir do qual é preciso continuar buscando novos desafios, e sem dúvida o MACT como um espaço cultural é um deles.

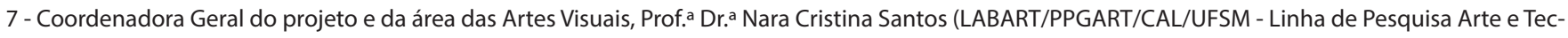
nologia)Grupo de Pesquisa CNPq - Arte e Tecnologia; coordenadora da área de Ciências, Prof. a Dr. ${ }^{\text {a }}$ Maria Rosa Chitolina (ENZITOX/PPGBTOX/CCNE/UFSM Linha de Pesquisa Enzimologia Toxicológica) Grupo de Pesquisa CNPq - Enzimologia Toxicológica e Neuroquímica. A partir de 2016, integra a coordenação da área de tecnologia a Prof. ${ }^{\text {a Dr. }}$ Juliana Kaizer Vizzotto (PPGI/CT/UFSM - Linha de Pesquisa de Linguagens de Programação e Banco de Dados) Grupo de Pesquisa CNPq - Linguagens de Programação e Banco de Dados.

8 - www.museuarteciencia.ufsm.br

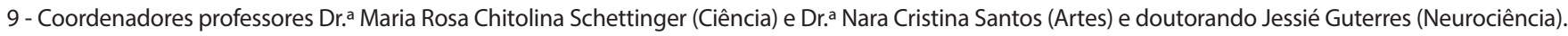

